\title{
Hadronic gas chiral phase transition within generalized chiral perturbation theory
}

\author{
J. R. Peláez* \\ Stanford Linear Accelerator Center, Stanford University, Stanford, California 94309
}

(Received 1 July 1998; published 13 November 1998)

\begin{abstract}
We study the temperature evolution of the $\langle\bar{q} q\rangle$ condensate below the chiral phase transition. The hadronic gas is described using a virial expansion within generalized chiral perturbation theory. In such a way, we can implement both the large or small chiral condensate scenarios and analyze the condensate dependence on the values of the lightest quark masses. [S0556-2821(98)02123-7]

PACS number(s): 12.39.Fe, 11.30.Rd, 21.65.+f
\end{abstract}

\section{INTRODUCTION}

The properties of QCD at finite temperature have raised considerable interest in the literature (see [1] and references therein). At low temperatures it seems that color is confined and chiral symmetry is spontaneously broken. However, from asymptotic freedom, it is expected that at high temperatures color will be liberated and chiral symmetry restored. It is a matter of intense debate whether there should be one or two phase transitions, at what temperatures they would occur, and what their nature would be.

Within the standard wisdom the quark chiral condensate $\langle 0|\bar{q} q| 0\rangle$ plays a central role in this problem, since it is assumed that chiral symmetry breaking is produced by a strong condensation of quark-antiquark pairs [2]. However, in recent years, this hypothesis has been questioned, opening the possibility of small, even vanishing, $\langle 0|\bar{q} q| 0\rangle$ scenarios [3-5]. (Note that we use $\langle 0|\bar{q} q| 0\rangle$ for the condensate at $T$ $=0$ and $\langle\bar{q} q\rangle$ in general.)

The evolution of the quark condensate with the temperature has indeed been addressed using several approaches. In general, the properties of $\langle\bar{q} q\rangle$ can be derived from a somewhat idealized dilute pion gas, which is commonly described using an effective Lagrangian formalism [6], as we will do here, or by means of finite temperature QCD sum rules [7]. In general, all these and other approaches [8] yield a rather consistent picture, although they usually have the large condensate assumption built in.

In this work we want to know how the actual value of $\langle 0|\bar{q} q| 0\rangle$, as well as the light quark masses, can modify the behavior of the chiral condensate, as for instance, with changes in the phase transition temperature. With that purpose, we will describe the pion gas by means of the virial expansion and using the interactions obtained from the chiral perturbation theory (ChPT) formalism [9], although allowing for a wide range of $\langle 0|\bar{q} q| 0\rangle$ values. Such a framework is usually referred to as generalized chiral perturbation theory (GChPT) [3,4]. It should be noticed that we will be dealing with two effects: First, at $T=0$, the size of $\langle 0|\bar{q} q| 0\rangle$, which may be different from the standard large value. Second, the

\footnotetext{
*On leave of absence from Departamento de Física Teórica, Universidad Complutense, 28040 Madrid, Spain. Electronic address: pelaez@slac.stanford.edu
}

evolution at finite temperature which is also changed through the modifications in the meson interactions due to the different scheme of the explicit chiral symmetry breaking. Our purpose is to study what the interplay is of these two effects.

The plan of the paper is as follows. In Sec. II we describe briefly the ChPT and GChPT formalisms, focusing on the relation between the quark and meson masses with the quark condensate. The next section is devoted to the virial expansion for the pion gas, where we introduce the temperature dependence. In Sec. IV we study the condensate dependence both on the temperature and the ratio of light quark masses, using the $\mathcal{O}\left(p^{4}\right)$ amplitudes of GChPT. Next, in Sec. V, we estimate the contributions from heavier states, and in Sec. VI we summarize our results.

\section{STANDARD AND GENERALIZED CHIRAL PERTURBATION THEORY}

When considering just three massless quark flavors, the QCD Lagrangian exhibits an $\mathrm{SU}(3)_{L} \times \mathrm{SU}(3)_{R}$ symmetry which, even neglecting particle masses, is not present in the physical spectrum. Instead, we observe an approximate $\mathrm{SU}_{L+R}(3)$ symmetry, which means that the $\mathrm{SU}(3)_{L-R}$ chiral group has to be spontaneously broken. According to the Goldstone Theorem, there should be eight massless Goldstone bosons (GB), which are identified with the pions, kaons and the eta. In a first approximation, these GB couple to the spontaneously broken currents with strength $F$ $\sim 90 \mathrm{MeV}$. These particles are so light compared with the typical hadronic scales, that they will dominate the hadronic dynamics at low energies or temperatures.

In order to describe the hadronic dynamics at low energies we can therefore use these fields to build an effective Lagrangian, made of the most general terms that respect the above symmetry breaking pattern. As we are interested in the low energy regime, the terms are organized according to their number of derivatives. It can be seen, by counting the powers of momenta of different diagrams, that it is possible to renormalize any calculation and obtain finite results order by order in the expansion [10]. We could also couple gauge fields, scalar and pseudoscalar sources, etc...., which would allow us to describe other processes. This whole approach is usually known as chiral perturbation theory (ChPT) [9].

\section{Explicit chiral symmetry breaking}

Up to the moment we have just considered the chiral limit. When quark masses are turned on, the GB become massive pseudo-GB and their masses can be obtained, generically, as 


$$
\begin{aligned}
& M_{\pi}^{2} \simeq 2 B_{0} \hat{m}+\mathcal{O}\left(m_{q}^{2}\right) \\
& M_{K}^{2} \simeq\left(\hat{m}+m_{s}\right) B_{0}+\mathcal{O}\left(m_{q}^{2}\right) \\
& M_{\eta}^{2} \simeq \frac{2}{3}\left(\hat{m}+2 m_{s}\right) B_{0}+\mathcal{O}\left(m_{q}^{2}\right),
\end{aligned}
$$

where $\hat{m}=\left(m_{u}+m_{d}\right) / 2$ (we will consider isospin as an exact symmetry) and $B_{0}$ and other coefficients that may appear at higher orders are to be determined phenomenologically. Throughout this work, the first one will play a very relevant role, since it has a very physical meaning: In the chiral limit, and up to a normalization factor, it is nothing but the chiral condensate; namely

$$
\langle 0|\bar{q} q| 0\rangle \equiv\langle 0|\bar{u} u+\bar{d} d| 0\rangle \stackrel{\hat{m} \rightarrow 0}{\longrightarrow}-2 F_{0}^{2} B_{0} .
$$

At this point two different approaches appear in the literature. The first one, still called ChPT [9], is to assume that the mass expansions in Eq. (1) are dominated by the $B_{0}$ term. Its origin can be traced to the Gell-Mann-Okubo (GMO) and Gell-Mann-Oakes-Renner (GOR) formulas, which, within the effective formalism, are obtained at first order by eliminating $B_{0}$ in Eqs. (1) and (2). This large condensate scenario usually requires $B_{0} \sim \mathcal{O}(1 \mathrm{GeV})$ and, apart from the GMO and GOR formulas, it is supported by several lattice calculations [11]. Within this framework, the quark masses count as $\mathcal{O}\left(p^{2}\right)$. The second approach, known as generalized chiral perturbation theory (GChPT) $[3,4]$, is nothing but considering the possibility that the $\mathcal{O}\left(m_{q}^{2}\right)$ terms could be of comparable size or even larger than the $B_{0}$ term. As a consequence, both the quark masses and $B_{0}$ count as $\mathcal{O}(p)$. This approach is supported by some deviations from the GoldbergerTreiman relation in $\pi N, K \Lambda$ and $K \Sigma$ [12] and some calculations using variationally improved perturbation theory or a relativistic many body approach [13].

Those two alternatives are usually compared with the spontaneous magnetization $\vec{M}$ of spin systems: On the one hand, ferromagnets present an ordered ground state where the magnetization spontaneously acquires an $\vec{M} \neq 0$ value. That would be analogous to the standard ChPT. On the other hand, in anti-ferromagnets the magnetization remains at $\vec{M}$ $=0$, which would be similar to the extreme case of GChPT where $B_{0}=0$. Note that, despite their difference, in both systems the spins are oriented in one preferred spatial direction and therefore the $S O(3)$ rotational symmetry is broken.

Back to our subject, it should be noticed that both approaches have the same terms in the Lagrangian, although they are organized differently, and their relative size is also changed. Indeed, it is possible to reobtain standard ChPT as a special case of GChPT.

At present, the experimental data does not exclude any of the two scenarios, although this question may be solved in a few years with an accurate measurement of $\pi \pi$ scattering lengths from the decay of $\pi^{+} \pi^{-}$atoms [14].

Thus, since we are interested in high temperature differences with the standard scenario, throughout this paper we will use the GChPT formalism. As usual, the pseudo-GB fields are grouped in an SU(3) matrix as follows:

$$
\Phi=\sqrt{2}\left(\begin{array}{ccc}
\frac{1}{\sqrt{2}} \pi^{0}+\frac{1}{\sqrt{6}} \eta & \pi^{+} & K^{+} \\
\pi^{-} & -\frac{1}{\sqrt{2}} \pi^{0}+\frac{1}{\sqrt{6}} \eta & K^{0} \\
K^{-} & \bar{K}^{0} & -\frac{2}{\sqrt{6}} \eta
\end{array}\right) .
$$

And then, with the GChPT power counting described above, the $\mathcal{O}\left(p^{2}\right)$ Lagrangian is usually written as

$$
\begin{aligned}
\widetilde{\mathcal{L}}^{(2)}= & \frac{4}{F^{2}}\left\{\operatorname{tr}\left(D_{\mu} U D^{\mu} U^{\dagger}\right)+2 B_{0} \operatorname{tr}\left(\mathcal{M}\left(U^{\dagger}+U\right)\right)\right. \\
& +A_{0} \operatorname{tr}\left(\mathcal{M} U^{\dagger} \mathcal{M} U^{\dagger}+\mathcal{M} U \mathcal{M} U\right) \\
& +Z_{0}^{S} \operatorname{tr}\left(\mathcal{M}\left(U+U^{\dagger}\right)\right)^{2}+Z_{0}^{P} \operatorname{tr}\left(\mathcal{M}\left(U-U^{\dagger}\right)\right)^{2} \\
& \left.+2 H_{0} \operatorname{tr}\left(\mathcal{M}^{2}\right)\right\},
\end{aligned}
$$

where $\mathcal{M}=\operatorname{diag}\left(\hat{m}, \hat{m}, m_{s}\right)$ is the quark mass matrix. In standard ChPT, only the two first terms are $\mathcal{O}\left(p^{2}\right)$, whereas the rest is counted as $\mathcal{O}\left(p^{4}\right)$. From the above Lagrangian we obtain the following meson masses:

$$
\begin{aligned}
M_{\pi}^{2}= & 2 \hat{m} B_{0}+4 \hat{m}^{2} A_{0}+4 \hat{m}\left(2 \hat{m}+m_{s}\right) Z_{0}^{S}, \\
M_{K}^{2}= & \left(\hat{m}+m_{s}\right) B_{0}+\left(\hat{m}+m_{s}\right)^{2} A_{0} \\
& +2\left(\hat{m}+m_{s}\right)\left(2 \hat{m}+m_{s}\right) Z_{0}^{S}, \\
M_{\eta}^{2}= & \frac{2}{3}\left(\hat{m}+2 m_{s}\right) B_{0}+\frac{4}{3}\left(\hat{m}^{2}+2 m_{s}^{2}\right) A_{0} \\
& +\frac{4}{3}\left(\hat{m}+2 m_{s}\right)\left(2 \hat{m}+m_{s}\right) Z_{0}^{S} \\
& +\frac{8}{3}\left(m_{s}-\hat{m}\right)^{2} Z_{0}^{P} .
\end{aligned}
$$

Comparing with Eq. (1), we have just added the $\mathcal{O}\left(m_{q}^{2}\right)$ terms. In the standard formalism, since only $B_{0}$ is present, it can be eliminated and one recovers, at $\mathcal{O}\left(p^{2}\right)$, the GMO and GOR relations. That is no longer possible in GChPT, although these relations will be recovered at higher orders. Of the three $\mathcal{O}\left(m_{q}^{2}\right)$ parameters there are two, $Z_{S}^{0}$ and $Z_{P}^{0}$, which violate the Zweig rule. They are expected to be small from large $N_{c}$ arguments and is usual to neglect their contribution, and so we will do likewise in most of what follows.

Note that, since the pion, kaon and eta mass values are known, then, changing the value of $B_{0}$ is nothing but changing the values of the quark masses. As a matter of fact, the 
ChPT relations are frequently used in the literature to obtain ratios of light quark masses (for a recent update, see [15] and references therein) or even to evaluate $\hat{m}$ itself. However, most of these works have used the standard ChPT formalism and have the large condensate assumption built in, so that their results would change if it was removed. Nevertheless, there are determinations of $m_{s}-m_{u}$, which do not rely on a large condensate value. For the sake of simplicity, and in order to facilitate the comparison with previous works [4], we will use the value $m_{s}-m_{u}=(184 \pm 32) \mathrm{MeV}$, given in [16]. That is,

$$
\hat{m}=\frac{184 \pm 32}{r-1} \mathrm{MeV}
$$

(There are other similar analyses in Ref. [17], whose results are all consistent with the previous relation.) As a consequence, the parameter that determines the relative size of the $\mathcal{O}\left(m_{q}\right)$ and $\mathcal{O}\left(m_{q}^{2}\right)$ terms is the quark mass ratio $r=m_{s} / \hat{m}$, which ranges in the interval

$$
r_{1} \equiv 2 \frac{M_{K}}{M_{\pi}}-1 \leqslant r \leqslant 2 \frac{M_{K}^{2}}{M_{\pi}^{2}}-1 \equiv r_{2}
$$

The upper limit corresponds to the extreme case of a very large $B_{0}$ condensate, whereas the second corresponds to $B_{0}$ $=0$. (Vacuum stability requires $B_{0}, A_{0}, Z_{0}^{S} \geqslant 0$.)

Of course, all these formulas are valid up to $\mathcal{O}\left(p^{2}\right)$. For the moment, we have restricted ourselves to the $\mathcal{O}\left(p^{2}\right)$ case since it already displays the features of GChPT which are relevant for this work. In section four we will state our results including higher order corrections, although we will just present the GChPT formulas without such a detailed introduction.

\section{The chiral condensate at zero temperature}

Using the GChPT Lagrangian in Eq. (4), the chiral condensate at $\mathcal{O}\left(p^{2}\right)$ is then given by

$$
\langle 0|\bar{q} q| 0\rangle=-2 F_{0}^{2}\left[B+\hat{m}\left(A_{0}+H_{0}\right)+\cdots\right],
$$

where $B=B_{0}+2\left(m_{s}+2 \hat{m}\right) Z_{0}^{S}$. In practice $B_{0}$ cannot be separated from $B$ by looking at quark masses alone, but we have already commented that $Z_{0}^{S}$ is expected to be very small, so that $B \sim B_{0}$. The parameter $H_{0}$ is associated with the contact term of two scalar sources, which does not contain meson fields. However, it is needed as a short distance counterterm, and it indeed depends on the renormalization conventions, which introduce some small ambiguity (see [9] for a discussion). Nevertheless, using QCD sum rules with a simple model for the spectral function, and keeping $F_{\pi}^{2} M_{\pi}^{2}$ fixed at its physical value, it has been found [18] that the chiral condensate can be described by

$$
\hat{m}\langle 0|\bar{q} q| 0\rangle \simeq-F_{\pi}^{2}\left[M_{\pi}^{2}-4 \hat{m}^{2} \Omega\right]
$$

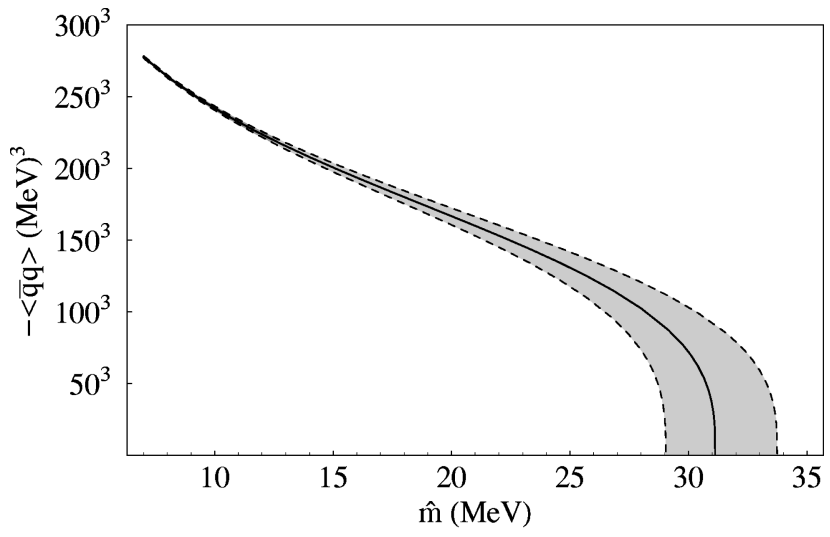

FIG. 1. The chiral condensate at zero temperature as a function of the light quark mass $\hat{m}$, Eq. (9). The continuous line corresponds to the central value of $\Omega$. The uncertainty due to its error is covered by the shaded area.

with $\Omega=4.7 \pm 0.7$. At $\mathcal{O}\left(p^{2}\right)$, the $\Omega$ parameter is nothing but $\left(A_{0}-H_{0}\right) / 2$. We will use the above equation to estimate the size of the quark condensate at $T=0$.

In Fig. 1 we show the dependence of the condensate with $\hat{m}$, for $\Omega=4.7$. Note that the plot starts at $\hat{m}=7 \mathrm{MeV}$, which is approximately the standard ChPT value. There, $\langle 0|\bar{q} q| 0\rangle \simeq-(280)^{3} \mathrm{MeV}^{3}$, and it decreases smoothly as $\hat{m}$ gets larger, until it vanishes around $\hat{m} \simeq 30 \mathrm{MeV}$. The shaded area between dashed lines covers the uncertainty in $\Omega$.

As a check of Eq. (9) we can see that it is consistent with previous estimates within the framework of standard ChPT [9], where

$$
\begin{aligned}
\hat{m}\langle 0|\bar{q} q| 0\rangle & =-F_{0}^{2} M_{\pi}^{2}\left[1+\frac{M_{\pi}^{2}}{32 \pi^{2} F_{\pi}^{2}}\left(4 \bar{h}_{1}+\bar{l}_{3}-1\right)\right] \\
& \equiv-F_{0}^{2} M_{\pi}^{2} \frac{1}{c}
\end{aligned}
$$

and the $\bar{h}_{1}$ and $\bar{l}_{3}$ parameters play a similar role as that of the GChPT $\mathcal{O}\left(p^{2}\right)$ parameters, although in Standard ChPT they appear at $\mathcal{O}\left(p^{4}\right)$. In that case, estimates based on a simple $\rho$ resonance model and the large $N_{c}$ limit yield $c=0.87$ and $c=0.90 \pm 0.05$, respectively [6]. If we introduce in Eq. (9) a value in the range $\hat{m}$ from 5 to $10 \mathrm{MeV}$, and take into account the fact that in standard ChPT $F_{\pi} / F_{0} \simeq 1.057$, we obtain $c=0.95 \pm 0.04$, which is a highly nontrivial check of Eq. (9). (Throughout this section we have neglected higher order logarithmic contributions that would yield corrections of the order of $1 \%$.)

\section{THE VIRIAL EXPANSION AND TEMPERATURE EFFECTS}

At low energies the free energy $z$ is dominated by the contributions from the lightest particles. Therefore, we can use the Euclidean form of the above Lagrangian [denoted $L(x)$ ] within the standard finite temperature functional $\mathrm{Eu}-$ 
clidean formalism. Hence, in the thermodynamic limit,

$$
z=-T \lim _{L \rightarrow \infty} \frac{1}{L^{3}} \int[d U] \exp \left(-\int_{L^{3} \times[0, T]} d^{4} x L(x)\right),
$$

where, as usual, the functional integration is over pion fields which are periodic in the Euclidean time, with period $\beta$ $=1 / T$ (see Ref. [6]). From the free energy we can derive any other thermodynamic property of our system, but let us first notice that since there is a spontaneously broken symmetry, even at $T=0$ there is some nonvanishing vacuum energy density $\epsilon_{0}$. As a consequence, the pressure is defined only from the temperature dependent part of the free energy, $P$ $\equiv \epsilon_{0}-z$.

The quark condensate is now obtained as the derivative of the free energy with respect to the quark mass. That is

$$
\langle\bar{q} q\rangle \equiv \frac{\partial z}{\partial \hat{m}}=\langle 0|\bar{q} q| 0\rangle-\frac{\partial P}{\partial \hat{m}},
$$

where we have used that at $T=0$ the condensate is nothing but the vacuum expectation value $\langle 0|\bar{q} q| 0\rangle \equiv \partial \epsilon_{0} / \partial \hat{m}$.

In this section we will just concentrate on how to obtain $\partial P / \partial \hat{m}$. For that purpose, one possibility is to calculate the free energy from the effective Lagrangian, as was done in [6] within standard ChPT. That method follows the very same philosophy of the chiral expansion, but is rather lengthy. In this paper we will make use of existing one loop calculations of elastic $\pi \pi$ scattering, together with the relativistic virial expansion of a pion gas $[19,20]$.

Let us then consider a gas made only of pions. This approximation seems reasonable as long as the temperatures remain sufficiently below the kaon threshold [6]. Within the virial formalism, the pressure can be expanded as follows

$$
P=3 T\left(\frac{M_{\pi} T}{2 \pi}\right)^{3 / 2} \sum_{k=1}^{\infty} B_{k} e^{-\beta M_{\pi^{k}}}=3 \frac{T}{\lambda^{3}} \sum_{k=1}^{\infty} B_{k}(T) \xi^{k}
$$

The factors of three come from the fact that we are neglecting isospin breaking effects. Thus, there are effectively three different species of particles, labeled according to their third isospin component, that behave identically with respect to strong forces. We have also defined $\lambda=\left(2 \pi / M_{\pi} T\right)^{1 / 2}$, which is the thermal pion wavelength. Note that the expansion parameter is the fugacity $\xi=e^{-\beta M_{\pi}}$. In a nonrelativistic framework, the expansion is usually performed using the definition $\xi=e^{\beta \mu}$, where $\mu$ would be the chemical potential. In contrast, in the relativistic case, there is a rest energy given by $M_{\pi}$, whose contribution to Eq. (11) is equivalent to a chemical potential $\mu=-M_{\pi}$ in a nonrelativistic description.

There is a closed expression for the virial coefficients of the free gas, which is

$$
B_{n}^{(0)}(T)=\frac{3}{n\left(M_{\pi} T\right)^{3 / 2}} \sqrt{\frac{2}{\pi}} \int_{0}^{\infty} d p p^{2} e^{-n \beta\left(E(p)-M_{\pi}\right)} .
$$

In order to deal with the interacting gas, we will just consider two particle interactions, which can be justified as long as the density remains small. In [6] it was shown that this is consistent with the three loop calculation in ChPT. In such case, it is enough to keep the two first terms of the virial expansion, whose coefficients will be given by [19]

$$
\begin{aligned}
B_{1}(T)= & B_{1}^{(0)}(T) \\
B_{2}(T)= & B_{2}^{(0)}(T)+\frac{4 e^{2 M_{\pi} / T}}{\left(2 \pi M_{\pi} T\right)^{3 / 2}} \int_{2 M_{\pi}}^{\infty} d E E^{2} K_{1}(E / T) \\
& \times\left(\sum_{I, J}(2 I+1)(2 J+1) \delta_{I J}(E)\right)
\end{aligned}
$$

where $K_{1}(x)$ is the modified Bessel function which behaves as $\sqrt{\pi / 2 x} e^{-x}$ when $x \rightarrow \infty$. It is important to notice that the only dynamical information we need are the phase shifts $\delta_{I J}$. As an estimate of the applicability of this approach, it was shown in [20] that the second order virial expansion yields less than a $1 \%$ error when applied to the free gas up to $T$ $\sim 250 \mathrm{MeV}$.

Let us finally remark that the high temperature behavior of the chiral condensate will be due to two different effects. First, the starting $T=0$ value, which may differ from the standard, large condensate, value. But, second, it also depends on how the mass dependence of the phase shifts has changed with respect to standard ChPT.

In the next section the phase shifts will we obtained using the existing GChPT calculations of the $\pi \pi$ elastic scattering amplitudes [4]. In later sections we will include contributions from particles more massive than pions.

\section{THE GENERAL SCENARIO}

\section{Higher orders in GChPT}

Within the standard ChPT it was shown in [6] that the $\mathcal{O}\left(p^{4}\right)$ contributions accelerate the melting of the chiral condensate, lowering the critical temperature. Our aim now is to include the equivalent corrections within GChPT. Unfortunately, we have already seen that the $\widetilde{\mathcal{L}}^{(2)}$ Lagrangian has more terms that the standard $\mathcal{L}^{(2)}$. That means that there are many more phenomenological parameters in the Lagrangian, which in many cases are not very well known. The situation gets even worse at higher orders. In general, the GChPT Lagrangian is built of terms like $[3,4]$

$$
\widetilde{\mathcal{L}}^{(d)}=\sum_{k+l+n} B_{0}^{n} \mathcal{L}_{(k, l)}, \quad \text { with } \quad \mathcal{L}_{(k, l)} \sim \mathcal{O}\left(p^{k} m_{q}^{l}\right)
$$

Indeed, we have already given $\widetilde{\mathcal{L}}^{(2)}$ in Eq. (4) and we found that some of the constants are not very well determined. For the complete expression of the $\mathcal{O}\left(p^{4}\right)$ Lagrangian we refer to [4]. For our purposes, there are several relevant modifications to our previous discussion: First, now there are three different decay constants $F_{\pi}, F_{K}$ and $F_{\eta}$. Second, neglecting Zweig rule violating parameters, the expressions for $M_{\pi}$ and $M_{K}$ in Eq. (5) are now modified to 


$$
\begin{aligned}
& \frac{F_{\pi}^{2}}{F^{2}} M_{\pi}^{2}=2 \hat{m} B_{0}+4 \hat{m}^{2} A_{0}+\frac{F_{\pi}^{2}}{F^{2}} \delta M_{\pi}^{2}, \\
& \frac{F_{K}^{2}}{F^{2}} M_{K}^{2}=\left(\hat{m}+m_{s}\right) B_{0}+\left(\hat{m}+m_{s}\right)^{2} A_{0}+\frac{F_{K}^{2}}{F^{2}} \delta M_{K}^{2},
\end{aligned}
$$

where $\delta M_{i}^{2}$ are higher order corrections and logarithmic terms [4], whose size is $\delta M_{i}^{2}<0.1 M_{i}^{2}$ [21] (see below). As a consequence, the range of allowed $r$ values is shifted upwards to

$$
r_{1}^{*} \equiv 2 \frac{F_{K} M_{K}}{F_{\pi} M_{\pi}}-1 \leqslant r \leqslant 2 \frac{\left(F_{K} M_{K}\right)^{2}}{\left(F_{\pi} M_{\pi}\right)^{2}}-1 \equiv r_{2}^{*} .
$$

With this modifications now $r_{1}^{*} \simeq 8$ and $r_{2}^{*}$ can be as large as 39.

Finally, there are also higher order corrections to the $T$ $=0$ condensate itself, which contain chiral logarithms. That means that we cannot simply say that $\Omega=\left(A_{0}+H_{0}\right) / 2$. Nevertheless we can still use the phenomenological parameter $\Omega=4.7 \pm 0.7$ in Eq. (9).

\section{The one loop $\pi \pi$ amplitude in GChPT}

Next, we need the $\pi^{+} \pi^{-} \rightarrow \pi^{0} \pi^{0}$ scattering amplitude itself. Although it has been calculated in GChPT up to two loops $[3,4]$, for our purposes it will be more than enough to consider the one loop result, which reads

$$
\begin{aligned}
A(s, t, u)= & \frac{\alpha}{3 F_{\pi}^{2}} M_{\pi}^{2}+\frac{\beta}{F_{\pi}^{2}}\left(s-\frac{4}{3} M_{\pi}^{2}\right)+\frac{\lambda_{1}}{F_{\pi}^{4}}\left(s-2 M_{\pi}^{2}\right)^{2} \\
& +\frac{\lambda_{1}}{F_{\pi}^{4}}\left[\left(t-2 M_{\pi}^{2}\right)^{2}+\left(u-2 M_{\pi}^{2}\right)^{2}\right] \\
& +\bar{J}_{(\alpha, \beta)}(s, t, u)
\end{aligned}
$$

where

$$
\begin{aligned}
\bar{J}_{(\alpha, \beta)}(s, t, u)= & \frac{1}{6 F_{\pi}^{4}}\left\{4\left[\frac{5}{6} \alpha M_{\pi}^{2}+\beta\left(s-\frac{4}{3} M_{\pi}^{2}\right)\right]^{2}\right. \\
& \left.-\left[\frac{2}{3} \alpha M_{\pi}^{2}-\beta\left(s-\frac{4}{3} M_{\pi}^{2}\right)\right]^{2}\right\} \bar{J}(s) \\
& +\frac{1}{12 F_{\pi}^{4}}\left\{3\left[\frac{2}{3} \alpha M_{\pi}^{2}-\beta\left(t-\frac{4}{3} M_{\pi}^{2}\right)\right]^{2}\right. \\
& \left.+\beta^{2}(s-u)\left(t-4 M_{\pi}^{2}\right)\right\} \bar{J}(t) \\
& +\frac{1}{12 F_{\pi}^{4}}\left\{3\left[\frac{2}{3} \alpha M_{\pi}^{2}+\beta\left(u-\frac{4}{3} M_{\pi}^{2}\right)\right]^{2}\right. \\
& \left.+\beta^{2}(s-t)\left(u-4 M_{\pi}^{2}\right)\right\} \bar{J}(u)
\end{aligned}
$$

and $\bar{J}$ is the standard one-loop integral [9].

In the literature, the values of the $\alpha, \beta, \lambda_{1}$ and $\lambda_{2}$ are fitted from experiment. However, in order to obtain the condensate dependence with the temperature, we need the derivative of the pressure with respect to $M_{\pi}$, and just a fitted value is not enough. Therefore, we also need to know the $M_{\pi}$ dependence of the parameters, and, if we want to study the effects of changing the light quark masses, we also need the dependence on $r$.

\section{Phenomenological parameters}

The actual expressions of the $\alpha, \beta$, parameters are rather complicated and involve many parameters from the GChPT Lagrangian, which frequently are not very well determined. In addition they contain chiral logarithms. It is therefore very convenient to expand $\alpha$ and $\beta$ in powers of quark masses, namely

$$
\alpha=\sum_{n=0}^{3} \alpha^{(n)}, \quad \beta=\sum_{n=0}^{3} \beta^{(n)}
$$

Notice that, in GChPT, since quark masses are considered as $\mathcal{O}(p)$, these expansions involve terms that count as odd powers of momenta.

The above expansions have been worked out in [4], and they are the following:

$$
\begin{aligned}
\alpha(r)= & 1+6 \frac{r_{2}^{*}-r}{r^{2}-1}-\frac{4}{r-1}\left(\frac{F_{K}^{2}}{F_{\pi}^{2}}-1\right) \\
& +18(2-r) \hat{\rho}_{1}-6 r \hat{\rho}_{2}+\alpha^{(2)}(r) \\
\beta(r)= & 1+\frac{2}{r-1}\left(\frac{F_{K}^{2}}{F_{\pi}^{2}}-1\right)+\beta^{(2)}(r),
\end{aligned}
$$

where in all the above equations we have neglected the Zweig rule violating parameters.

Let us now try to estimate the size of the different terms in the $\alpha$ and $\beta$ expansions. Let us then look back at the allowed values of $r$, Eq. (18). The relevant point for our discussion is that now, even with the lowest value $r=8$, we obtain, using Eq. (6), that $\hat{m} \leqslant(26 \pm 4.6) \mathrm{MeV}$. Therefore we can estimate that the terms coming from $B_{0}$ and $A_{0}$ should be $\mathcal{O}(1)$, those from $\widetilde{\mathcal{L}}^{(3)}$ should be $\mathcal{O}(10 \%)$ and those from $\mathcal{L}_{(2,2)}$ and $\mathcal{L}_{(0,4)}$ should at most reach the $1 \%$ level. Consequently, we will neglect the $\alpha^{(2)}$ and $\beta^{(2)}$ effects. The only parameters that remain undetermined are the $\hat{\rho}_{1,2}$, which contribute to $\alpha^{(1)}$. However, from a dimensional analysis [4], their magnitude can be naively estimated as $\left|\hat{\rho}_{i}\right| \simeq(0.4 \pm 0.2) /(r-1)^{3}$. Their dependence on the actual value of $M_{\pi}$ (needed for the numerical derivation) seems very weak. In our calculations we will take them first as zero and then we will include them in the uncertainty.

Concerning $\lambda_{1}$ and $\lambda_{2}$, they come only from the terms in $\mathcal{L}_{(4,0)}$, which do not contain explicit chiral symmetry breaking. They are given by 


$$
\begin{aligned}
\lambda_{1}= & \lambda_{1}^{(0)}=4\left(2 L_{1}^{r}(\mu)+L_{3}\right)-\frac{1}{48 \pi^{2}}\left\{\log \frac{M_{\pi}^{2}}{\mu^{2}}\right. \\
& \left.+\frac{1}{8} \log \frac{M_{K}^{2}}{\mu^{2}}+\frac{35}{24}\right\}, \\
\lambda_{2}= & \lambda_{2}^{(0)}=4 L_{2}^{r}(\mu)-\frac{1}{48 \pi^{2}}\left\{\log \frac{M_{\pi}^{2}}{\mu^{2}}\right. \\
& \left.+\frac{1}{8} \log \frac{M_{K}^{2}}{\mu^{2}}+\frac{23}{24}\right\} .
\end{aligned}
$$

It can be seen that these parameters do not carry any $r$ dependence. For definiteness, we will use for them the values obtained in [4]:

$$
\lambda_{1}=(-5.3 \pm 2.5) \times 10^{-3} ; \quad \lambda_{2}=(9.7 \pm 1.0) \times 10^{-3},
$$

which are consistent with other determinations in the standard framework.

The values of $\alpha$ and $\beta$ depend on whether there is actually a large or small condensate at $T=0$, and we will use their $r$ dependence to reproduce different scenarios. For illustrative purposes, let us recall that in the standard formalism both $\alpha$ and $\beta$ are slightly bigger than one and $r \simeq 26$. In contrast, the low condensate alternative seems to prefer $\alpha \simeq 2$ and $r \simeq 10$ [4].

\section{Phase shifts}

For the virial expansion we need the phase shifts of definite isospin and angular momentum channels. At lowest order, they are defined as follows (see [22] for a discussion on this subject)

$$
\tan \delta_{I J}(s)=\sigma(s) \operatorname{Re}\left(t_{I J}(s)\right),
$$

where $\sigma(s)=\sqrt{1-4 M_{\pi}^{2} / s}$. The partial waves $t_{I, J}$ are obtained from the isospin amplitudes

$$
\begin{aligned}
& T_{0}(s, t, u)=3 A(s, t, u)+A(t, s, u)+A(u, t, s), \\
& T_{1}(s, t, u)=A(t, s, u)-A(u, t, s), \\
& T_{2}(s, t, u)=A(t, s, u)+A(u, t, s),
\end{aligned}
$$

by means of

$$
t_{I J}=\frac{1}{64 \pi} \int_{-1}^{1} d(\cos \theta) P_{J}(\cos \theta) T_{I}(s, t, u),
$$

where $P_{I}$ is the corresponding Legendre polynomial. In our calculations we have just used the lowest angular momentum for each isospin channel, namely $(I, J)=(0,0),(1,1)$ and $(2,0)$. For all means and purposes, they dominate the low energy pion interactions.

\section{The calculation of $\partial P / \partial \hat{m}$}

We have then used the above phase shifts with the second order virial expansion. In order to obtain the condensate, Eq. (12), we then need $\partial P / \partial \hat{m}$, which can be obtained using

$$
\frac{\partial P}{\partial \hat{m}}=\frac{\partial P}{\partial M_{\pi}} \frac{\partial M_{\pi}}{\partial \hat{m}}+\frac{\partial P}{\partial M_{K}} \frac{\partial M_{K}}{\partial \hat{m}}+\frac{\partial P}{\partial F_{\pi}} \frac{\partial F_{\pi}}{\partial \hat{m}}+\frac{\partial P}{\partial F_{K}} \frac{\partial F_{K}}{\partial \hat{m}} .
$$

Naively one just expects the first term, but let us remember that $M_{K}, F_{\pi}$ and $F_{K}$ are $\hat{m}$ dependent and they appear in the amplitude either directly or indirectly through $\alpha, \beta, \lambda_{1}$ and $\lambda_{2}$. That problem was carefully avoided in [6] by using $\mathrm{SU}(2)$ standard ChPT and only using $F$ in the free energy expansion.

Of course, only $M_{\pi}$ appears in the fugacity, or in the free gas virial coefficients and thus we expect the three last terms in Eq. (28) to be much smaller than the first. Indeed, within the range of $r$ and $T$ that we are interested in, we have found that the term due to the appearance of $M_{K}$ in the amplitude is smaller than $1 \%$ and we have neglected it. In contrast, $F_{\pi}$ and $F_{K}$ together generate contributions of the order of $5 \%$, and therefore they have been included in our calculations.

The derivative of the pressure with respect to $M_{\pi}, F_{\pi}$ and $F_{K}$ have been performed numerically, with an increment of $0.1 \mathrm{MeV}$. For instance, the value of the pressure is first calculated with the real $M_{\pi}$ and then with $M_{\pi}-\delta M_{\pi}$, including a change in the chiral parameters, following Eqs. (22) and (23). A similar procedure is followed for $F_{\pi}$ and $F_{K}$.

In our calculations we have used

$$
\begin{aligned}
& \frac{\partial M_{\pi}}{\partial \hat{m}} \simeq \frac{M_{\pi}}{2 \hat{m}}\left[1+2 \frac{r_{2}^{*}-r}{r^{2}-1}\right], \\
& \frac{\partial M_{K}}{\partial \hat{m}} \simeq \frac{M_{\pi}^{2}}{4 \hat{m} M_{K}} \frac{r\left(2 r_{2}^{*}-r\right)-1}{r^{2}-1}, \\
& \frac{\partial F_{\pi}}{\partial \hat{m}} \simeq \frac{F_{\pi}}{\hat{m}\left[(r-1)+\left(F_{K}^{2} / F_{\pi}^{2}-1\right)\right]}\left(\frac{F_{K}^{2}}{F_{\pi}^{2}}-1\right), \\
& \frac{\partial F_{\pi}}{\partial \hat{m}} \simeq \frac{r-1}{2} \frac{\partial F_{\pi}}{\partial \hat{m}},
\end{aligned}
$$

which we have obtained from Eqs. (17) and from [4]. There are, of course, corrections, but their effects on the final results are again less than $1 \%$.

\section{Results}

As we have already commented, the virial expansion can be trusted only at low temperatures, mostly, due to the fact that above $\sim 150 \mathrm{MeV}$ the contributions from other more massive particles becomes relevant. These effects will be studied in the next section and we will see that they tend to lower the critical temperature, which is therefore more favor- 


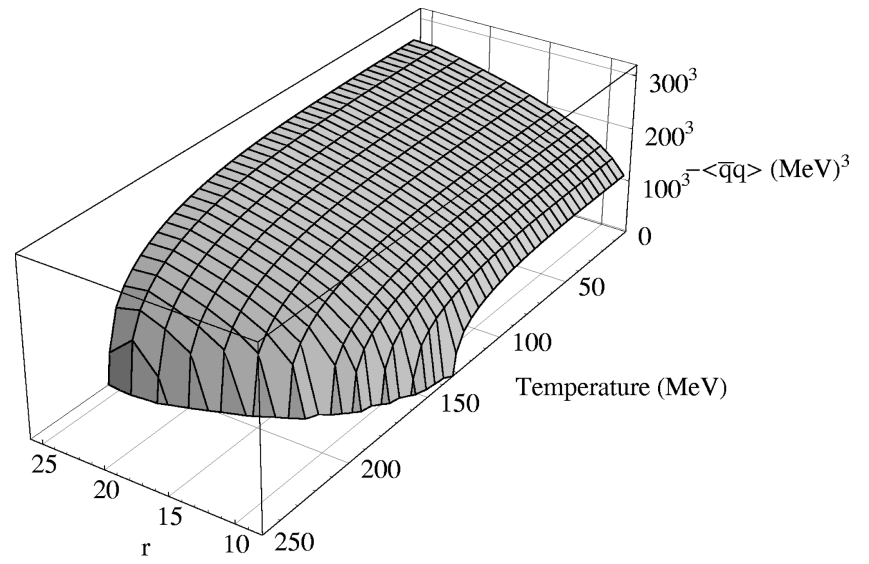

FIG. 2. Quark condensate versus temperature and $r$ for $\Omega$ $=4.7$.

able for our approach. For the moment, if we give in our figures results for higher temperatures, they should be interpreted with great care as a qualitative behavior or, for instance, as a tendency towards symmetry restoration. Nevertheless, comparing between different figures could also illustrate what is the qualitative effect of a change in the parameters.

Thus, in Fig. 2 we have shown the dependence of the chiral condensate with the temperature and for a light quark ratio in the range $8 \leqslant r \leqslant 26$. For $\Omega$ we have used the central value 4.7. Although the actual points at which $\langle\bar{q} q\rangle=0$ are just gross estimates, we can see that lowering $r$ yields a systematic decrease in the chiral phase transition temperature.

Indeed, $T_{C}$ seems to be above $200 \mathrm{MeV}$ for $r \geqslant 20$ going down to $130 \mathrm{MeV}$ around $r=8$. Note that for the latter temperature our approximations can become quite reliable. As we have already seen, smaller values of $r$ are forbidden to ensure vacuum stability.

The previous results have been obtained using the central values of all parameters. In Fig. 3(a) we show what happens if we take into account the uncertainty in $\Omega$ and thus, we plot the temperature dependence for the two extreme cases, $r$ $=26$ and $r=8$. The former, which corresponds to the upper curve, is almost insensitive to this variation. It corresponds to the standard formalism, where the value of the chiral condensate is largely dominated by the $\mathcal{O}(m)$ term and, consistently, changes in the other terms are almost negligible. On the other curve, which is associated with the lowest condensate scenario, the effect of this error is translated to a 10 to $15 \%$ change in $T_{C}$, at most.

In Fig. 3(b) we show the uncertainties associated with all the parameters that appear in the scattering amplitude. In the shaded areas, we have taken into account all the effects of changing $\lambda_{1}, \lambda_{2}, \hat{\rho}_{1}$ and $\hat{\rho}_{1}$. In addition we have also let the pion and kaon masses vary between their values for the neutral or scalar particle. Note that in the case of the pion mass, such a change also affects the coefficients of the virial expansion and the fugacity. Finally, we have included the uncertainty in $F_{K} / F_{\pi}=1.22 \pm 0.01$ and we have let $F_{\pi}$ change between 92.4 and $93.2 \mathrm{MeV}$ which are two values currently cited in the literature. Both $M_{\pi}$ and $F_{\pi}$ do also appear in the expression of the $T=0$ chiral condensate. All in all, the overall uncertainty in $T_{C}$ due to these parameters seems to be of the order of $\pm 5 \mathrm{MeV}$ at $r=26$ and $\pm 3 \mathrm{MeV}$ at $r=8$. Since we have just simply added the different errors, we consider these numbers as a conservative estimate.

\section{OTHER MASSIVE PARTICLES}

In this section we will consider the effect of adding heavier particles to our pion gas. We will be following closely the approach of Gerber and Leutwyler [6] with slight modifications to implement also the low condensate scenario.

The density of massive states should be exponentially suppressed by Boltzmann factors $\exp \left(-M_{i} / T\right)$. Their two body interactions and their interactions with pions will therefore contribute to the second virial coefficient, but suppressed by an $\exp \left[-\left(M_{i}+M_{j}\right) / T\right]$ factor. Hence, we will treat those heavier particles in the free gas approximation. In such case, we have an additional contribution to the pressure, which is given by

$$
\Delta P=-\sum_{i} \frac{g_{i} T}{2 \pi^{2}} \int_{0}^{\infty} d p p^{2} \log \left[1-e^{-\sqrt{p^{2}+M_{i}^{2}} / T}\right]
$$

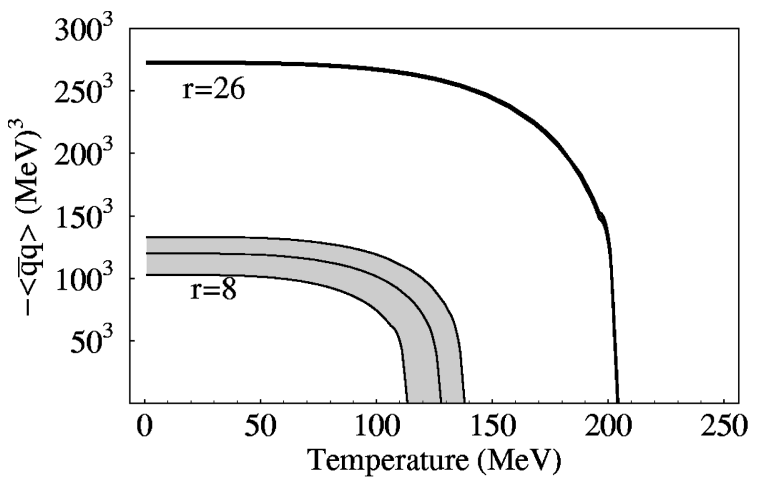

FIG. 3. (a) Estimate of the errors in the $\langle\bar{q} q\rangle$ evolution due to the uncertainty in $\Omega$. (b) Uncertainties in the parameters that appear in the $\pi \pi$ phase shifts, added linearly. 
where $g_{i}$ is the state degeneracy of a state with mass $M_{i}$ [that was the factor of 3 in Eq. (13)]. Note that, since we will be dealing with temperatures much smaller than the first hadronic fermions, it makes sense just to use Bose statistics. The above formula is only meaningful at low temperatures, since as we increase the temperature, the mean distance between massive states shrinks and the dilute gas approximation is no longer valid. In Ref. [9] it was estimated that the model is valid up to temperatures on the order of $150 \mathrm{MeV}$, although it "rapidly deteriorates" for higher temperatures.

Back to the condensate, and in view of Eq. (12), the new contributions are of the form

$$
\Delta\langle\bar{q} q\rangle=-\sum_{i} \frac{\partial \Delta P}{\partial M_{i}} \frac{\partial M_{i}}{\partial \hat{m}}
$$

and therefore

$$
\begin{aligned}
\Delta\langle\bar{q} q\rangle= & \frac{1}{2 \pi^{2}} \sum_{i} g_{i} M_{i} \frac{\partial M_{i}}{\partial \hat{m}} \\
& \times \int_{0}^{\infty} d p \frac{p^{2}}{\sqrt{p^{2}+M_{i}^{2}}} \frac{1}{e^{\sqrt{p^{2}+M_{i}^{2}} / T}-1} .
\end{aligned}
$$

Thus, we only have to estimate the value of $\partial M_{i} / \partial \hat{m}$. Naively, one would expect that the contribution $\hat{m}$ to a hadron mass would be roughly proportional to the number $N_{i}$ of $u$ and $d$ quarks it contains. That estimate seemed quite appropriate in the standard framework [6]. We now have to check that it is also the case in GChPT.

Let us then go back to Eqs. (5), since to get rough estimates it is enough to work at $\mathcal{O}\left(p^{2}\right)$. As usual, we neglect the $Z_{0}^{S}$ and $Z_{0}^{P}$ parameters. Then, we obtain the following derivatives

$$
\begin{aligned}
& \frac{\partial M_{K}}{\partial \hat{m}} \simeq \frac{M_{\pi}^{2}}{4 \hat{m} M_{K}} \frac{r\left(2 r_{2}-r\right)-1}{r^{2}-1}, \\
& \frac{\partial M_{\eta}}{\partial \hat{m}} \simeq \frac{M_{\pi}^{2}}{6 \hat{m} M_{\eta}}\left[1+2 \frac{r_{2}-r}{r^{2}-1}\right] .
\end{aligned}
$$

We can reproduce the standard scenario with $r=26$, which yields $\hat{m} \simeq 7.4 \pm 1.3 \mathrm{MeV}$ using Eq. (6). In such case, we find $\partial M_{K} / \partial \hat{m} \simeq 1.3 \pm 0.2$, which is in very good agreement with a rough estimate of 1 . We also find $\partial M_{\eta} / \partial \hat{m} \simeq 0.8$ \pm 0.1 , again consistent with the naive estimate of $2 / 3$. In any case, it seems that $\partial M_{i} / \partial \hat{m}=N_{i}$ is a small underestimate of the actual values of the standard scenario, as it was already pointed out in [6]. Thus, in that work they considered that the range from $N_{i}$ to $2 N_{i}$ was a "fair representation" of the uncertainty in $\partial M_{i} / \partial \hat{m}$.

However, if we set $r=8$, which corresponds to the lowest allowed $T=0$ condensate, we find $\partial M_{K} / \partial \hat{m} \simeq 2.0 \pm 0.4$ and $\partial M_{\eta} / \partial \hat{m} \simeq 0.36 \pm 0.06$. Again, the order of magnitude is correct, although within a factor of 2 . We will therefore use the

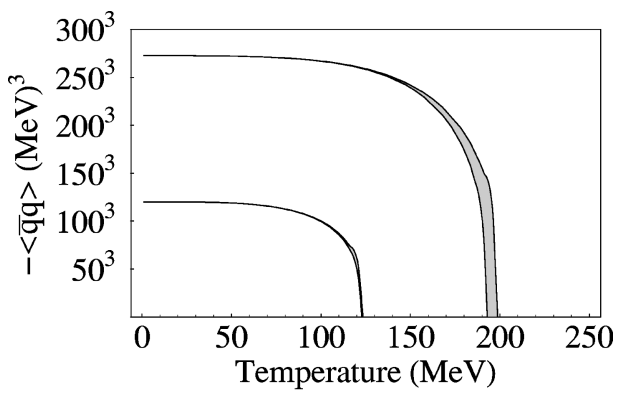

FIG. 4. The evolution of the chiral condensate when we include corrections from a free gas of particles more massive than the pion. The shadowed regions cover the uncertainties in $\partial M_{P} / \partial \hat{m}$ described in the text. These contributions always tend to lower the critical temperature.

estimates in Eq. (33) for the kaon and the eta, instead of $\partial M_{i} / \partial \hat{m}=N_{i}$. Those are the states that will contribute more at low temperatures. For the rest, we will assume the uncertainty in $\partial M_{i} / \partial \hat{m}=N_{i}$ to be from $N_{i} / 2$ to $2 N_{i}$.

Thus, in Fig. 4 we show the results when the massive states are taken into account. We have considered in Eq. (31) all particles containing $u$ and $d$ quarks up to $1300 \mathrm{MeV}$ and we have taken the central values of all the other parameters. The dominant contributions are, of course, those of the kaons, the eta, the rho and the omega. The shaded areas cover the uncertainty in $\partial M_{i} / \partial \hat{m}$ that we have just described. Obviously, the net effect is biggest for the standard scenario, since the critical temperature is higher, where $T_{C}$ is decreased down to 190 to $200 \mathrm{MeV}$. This result, although it has been obtained within the generalized formalism, reproduces very nicely the standard ChPT estimate given in [6].

Indeed, the $r$ dependence is given in Fig. 5 where we plot the evolution of the chiral condensate both with $T$ and $r$, for the central values of all the parameters, but also including the contributions from massive states. Note that, for the extreme case when $r=8$, the decrease is of the order of $5 \mathrm{MeV}$, down to around $125 \mathrm{MeV}$.

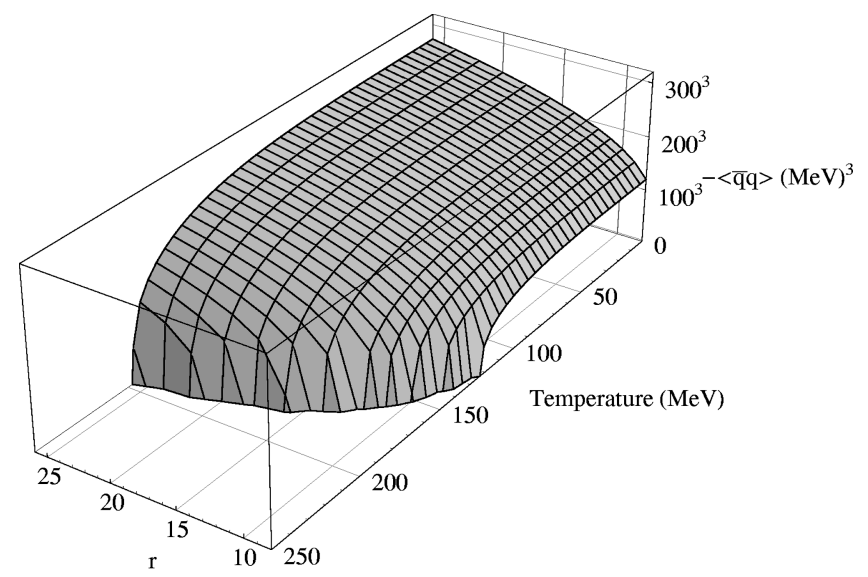

FIG. 5. The evolution of the chiral condensate when we include corrections in the pion gas from heavier states, as a function of the temperature and the quark mass ratio $r$ (using the central values of all parameters and estimates). 


\section{CONCLUSIONS}

In this work we have studied the generalized scenario of chiral symmetry breaking, either with a large or a small $T$ $=0$ condensate. For that purpose we have described a pion gas by means of the virial expansion, whose coefficients have been calculated using the amplitudes obtained within $\mathcal{O}\left(p^{4}\right)$ generalized chiral perturbation theory.

We have also added a crude estimate of contributions from particles heavier than the pion, in a free gas approximation, which can be justified at low temperatures. The effect of these particles is always to decrease the temperature of chiral restoration. Their net effect is to lower $T_{C}$ by 10 to $20 \mathrm{MeV}$ in the standard scenario, and by around $5 \mathrm{MeV}$ when the $T=0$ chiral condensate is smallest.

From our results, it seems that the chiral phase transition in a pure pionic gas may occur at energies as low as 125 $\mathrm{MeV}$ in the lowest possible $T=0$ condensate scenario. The main source of uncertainty is the fact that within the generalized approach many parameters still remain undetermined. In the worst case, which again corresponds to the lowest condensate and lowest $T_{C}$, it can be estimated at about $20 \%$. For the standard case of a large condensate, we recover previous estimates of $T_{C} \simeq 190 \mathrm{MeV}$.
In conclusion, we have found that the value of $\sim 190 \mathrm{MeV}$ for the critical temperature obtained from standard chiral perturbation theory can be seen as an upper bound if we were to include $\mathcal{O}\left(m_{q}^{2}\right)$ corrections in the mass terms, in addition to the standard condensate contribution. The effects of these corrections always lower the critical temperature, which, all together, could be as low as $125 \mathrm{MeV}$ with a $20 \%$ uncertainty for the lowest condensate scenario.

\section{ACKNOWLEDGMENTS}

I am especially indebted to A. Dobado for introducing me to the subject of pion gas thermodynamics, as well as J. Stern for explaining to me the present status of some error estimates, D. Espriu for several comments and suggestions, and J. Wells for a careful reading of the manuscript. I would also like to thank the Theory Group at SLAC for their kind hospitality and the Spanish Ministerio de Educación y Cultura for financial support. This work has been partially supported by the Spanish CICYT under contract AEN93-0776. This research was supported by the Department of Energy under contract DE-AC03-76SF00515.
[1] E. V. Shuryak, The QCD Vacuum, Hadrons and the Superdense Matter (World Scientific, Singapore, 1988); H. MeyerOrtmanns, Rev. Mod. Phys. 68, 473 (1996).

[2] M. Gell-Mann, Caltech Report CTSL-20 (1961); S. Okubo, Prog. Theor. Phys. 27, 949 (1962); M. Gell-Mann, R. J. Oakes, and B. Renner, Phys. Rev. 175, 2195 (1968).

[3] N. H. Fuchs, H. Sazdjian, and J. Stern, Phys. Lett. B 269, 183 (1991).

[4] J. Stern, H. Sazdjian, and N. H. Fuchs, Phys. Rev. D 47, 3814 (1993); M. Knecht, B. Moussallam, J. Stern, and N. H. Fuchs, Nucl. Phys. B457, 513 (1995).

[5] J. Stern, hep-ph/9801282.

[6] P. Gerber and H. Leutwyler, Nucl. Phys. B321, 387 (1989).

[7] M. Dey, V. L. Eletsky, and B. L. Ioffe, Phys. Lett. B 252, 620 (1990); V. L. Eletsky and B. L. Ioffe, Phys. Rev. D 47, 3083 (1993); 51, 2371 (1995); T. Hatsuda, Y. Koike, and S. H. Lee, Nucl. Phys. B394, 221 (1993).

[8] G. Chanfray, M. Ericson, and J. Wambach, Phys. Lett. B 388, 673 (1996).

[9] J. Gasser and H. Leutwyler, Ann. Phys. (N.Y.) 158, 142 (1984); Nucl. Phys. B250, 465 (1985).

[10] S. Weinberg, Phys. Rev. 166, 1568 (1968); Physica A 96, 327 (1979)

[11] S. Aoki et al. (CP-PACS), Nucl. Phys. B (Proc. Suppl.) 60A,
14 (1998); V. Giménez et al., hep-lat/9801028.

[12] N. H. Fuchs, H. Sazdjian, and J. Stern, Phys. Lett. B 238, 380 (1990).

[13] G. Arvanitis et al., Phys. Lett. B 390, 385 (1997); J. L. Kneur, Phys. Rev. D 57, 2785 (1998); Nucl. Phys. B (Proc. Suppl.) 64, 296 (1998); A. Szczepaniak et al., Phys. Rev. Lett. 76, 2011 (1996).

[14] B. Adeva et al., "Lifetime measurement of $\pi^{+} \pi^{-}$atoms to test low-energy QCD predictions,"' proposal to the SPSLC, CERN/SPSLC 95-1, SPSLC/P 284, Geneva, 1995.

[15] H. Leutwyler, Phys. Lett. B 378, 313 (1996).

[16] M. Jamin and M. Münz, Z. Phys. C 66, 633 (1995).

[17] K. G. Chetykin et al., Phys. Rev. D 51, 5090 (1995); S. Narison, Phys. Lett. B 358, 113 (1995).

[18] J. Stern, Nucl. Phys. B (Proc. Suppl.) 64, 232 (1998).

[19] R. Dashen, S. K. Ma, and H. J. Berstein, Phys. Rev. 187, 345 (1969).

[20] A. Dobado and J. R. Peláez, SLAC-PUB-7857, hep-ph/9806416.

[21] J. Stern, to appear in the Proceedings of the Workshop on Chiral Dynamics 1997, Mainz, Germany, 1997, hep-ph/9712438.

[22] J. Gasser and U. G. Meißner, Nucl. Phys. B357, 90 (1991); Phys. Lett. B 258, 219 (1991); A. Dobado and J. R. Peláez, Z. Phys. C 57, 501 (1993). 